\title{
Pax6, Tbr2, and Tbr1 Are Expressed Sequentially by Radial Glia, Intermediate Progenitor Cells, and Postmitotic Neurons in Developing Neocortex
}

\author{
Chris Englund, ${ }^{1}$ Andy Fink, ${ }^{1}$ Charmaine Lau, ${ }^{1}$ Diane Pham, ${ }^{1}$ Ray A. M. Daza, ${ }^{1}$ Alessandro Bulfone, ${ }^{2}$ Tom Kowalczyk, ${ }^{1}$ \\ and Robert F. Hevner ${ }^{1}$ \\ ${ }^{1}$ Department of Pathology, University of Washington, Seattle, Washington 98195-7470, and 2 Stem Cell Research Institute, Istituto Scientifico San Raffaele, \\ 20132 Milan, Italy
}

The developing neocortex contains two types of progenitor cells for glutamatergic, pyramidal-projection neurons. The first type, radial glia, produce neurons and glia, divide at the ventricular surface, and express Pax6, a homeodomain transcription factor. The second type, intermediate progenitor cells, are derived from radial glia, produce only neurons, and divide away from the ventricular surface. Here we show that the transition from radial glia to intermediate progenitor cell is associated with upregulation of Tbr2, a T-domain transcription factor, and downregulation of Pax6. Accordingly, Tbr2 expression in progenitor compartments (the subventricular zone and ventricular zone) rises and falls with cortical plate neurogenesis. The subsequent transition from intermediate progenitor cell to postmitotic neuron is marked by downregulation of Tbr2 and upregulation of Tbrl, another T-domain transcription factor. These findings delineate the transcription factor sequence Pax $6 \rightarrow \mathrm{Tbr} 2 \rightarrow \mathrm{Tbr} 1$ in the differentiation of radial glia $\rightarrow$ intermediate progenitor cell $\rightarrow$ postmitotic projection neuron. This transcription factor sequence is modified in preplate neurons, in which Tbr 2 is transiently coexpressed with Tbr 1 , and in the direct differentiation pathway from radial glia $\rightarrow$ postmitotic projection neuron, in which Tbr 2 is expressed briefly or not at all.

Key words: cerebral cortex; eomesodermin; neurogenesis; projection neurons; subventricular zone; ventricular zone

\section{Introduction}

Glutamatergic neocortical neurons are produced during embryonic development from two distinct types of progenitor cells. The first type, radial glia, are well known structural elements that, in the past few years, have also been recognized as neuronal and glial progenitors (Malatesta et al., 2000; Miyata et al., 2001; Noctor et al., 2001; Heins et al., 2002). The second type, intermediate progenitor cells (IPCs), were characterized recently as neuronal progenitors that divide away from the ventricular surface (Haubensak et al., 2004; Miyata et al., 2004; Noctor et al., 2004). IPCs are generated from radial glia, proliferate for one or two mitotic cycles, and produce neurons only. Whereas radial glia divide at the ventricular (apical) surface, IPCs divide at non-surface positions within the ventricular zone (VZ) and subventricular zone (SVZ). Thus, IPCs are also known as "non-surface-dividing (NSdiv) cells" or "basal progenitors."

The observation that IPCs are neurogenic has raised fundamental questions about their functional significance, their con-

Received July 16, 2004; revised Nov. 18, 2004; accepted Nov. 20, 2004.

This work was supported by National Institutes of Health Grant K02 NSO45018 and by the Edward Mallinckrodt, Jr. Foundation. R.F.H. is the 40th Mallinckrodt Scholar and the recipient of the Shaw Professorship in Neuropathology. We thank Dr. S. Anderson for comments on a previous version of this manuscript and M. Nivison for proofreading.

Correspondence should be addressed to Dr. Robert Hevner, Harborview Pathology, Box 359791, 325 Ninth Avenue, Seattle, WA 98104. E-mail: rhevner@u.washington.edu.

DOI:10.1523/JNEUROSCI.2899-04.2005

Copyright $\odot 2005$ Society for Neuroscience $\quad$ 0270-6474/05/250247-05\$15.00/0 tributions to different cortical layers, and their molecular properties. Evidence suggests that IPCs contribute substantial numbers of neurons for most or all layers. Smart (1973) documented that non-surface mitoses are present throughout corticogenesis, mainly in the SVZ, but also in the VZ and intermediate zone (IZ). During early neurogenesis when lower layers are produced, NS-div cells account for $>50 \%$ of neuron-producing mitoses (Haubensak et al., 2004). During mid-neurogenesis, NS-div cells account for between 32 and $100 \%$ of neuron production (Miyata et al., 2004). In late neurogenesis, many upper-layer neurons appear to be derived from SVZ progenitors (Tarabykin et al., 2001). IPCs also appear to have unique molecular expression profiles. Unlike radial glia, progenitors in the SVZ express Svet 1 but do not express Pax6, GLAST, or hGFAP-Cre transgene (Götz et al., 1998; Tarabykin et al., 2001; Malatesta et al., 2003).

In the present study, we show that Tbr2, a T-domain transcription factor (TF) in the developing brain (Bulfone et al., 1999), is highly expressed in neuronal progenitors in the SVZ and basal VZ of neocortex, consistent with IPCs. We further demonstrate that Pax6 is substantially downregulated in most Tbr2 ${ }^{+}$ cells, and that, in turn, Tbr2 is reduced to undetectable levels in postmitotic projection neurons, which instead express Tbr1, another T-domain TF (Bulfone et al., 1995; Hevner et al., 2001). Our findings identify Tbr2 as a molecular marker that is strongly expressed by NS-div cells and delineate the TF sequence Pax6 $\rightarrow$ Tbr2 $\rightarrow$ Tbr1 in the differentiation from radial glia $\rightarrow$ IPC $\rightarrow$ postmitotic projection neuron. 


\section{Materials and Methods}

Animals and tissue preparation. B6 mice (The Jackson Laboratory, Bar Harbor, ME) were used under a protocol approved by the Institutional Animal Care and Use Committee at the University of Washington. Procedures were described previously (Hevner et al., 2004). Briefly, timedpregnant dams [plug day was embryonic day 0.5 (E0.5)] received bromodeoxyuridine (BrdU) by intraperitoneal injection $(40 \mathrm{mg} / \mathrm{kg}) 30 \mathrm{~min}$ before cervical dislocation under Avertin anesthesia. Embryos were removed and used for cryosectioning or cell cultures. For cryosectioning, heads were fixed by immersion in cold buffered $4 \%$ paraformaldehyde for $16-20 \mathrm{hr}$ at $4^{\circ} \mathrm{C}$, cryoprotected with sucrose, and frozen in OCT compound (Sakura Finetek, Torrance, CA). Sections were cut at 10-12 $\mu \mathrm{m}$, air dried, and stored at $-80^{\circ} \mathrm{C}$.

Primary cortical cell cultures. Neocortex was pooled from four or eight embryos and dissociated with $0.05 \%$ trypsin as described previously (Xu et al., 2004). Cells were resuspended in DMEM containing $10 \%$ fetal bovine serum (Invitrogen, Carlsbad, CA) and plated in eight-well chamber slides (Nunc LabTek, Rochester, NY), which were previously coated with poly-D-lysine $(10 \mu \mathrm{g} / \mathrm{ml})$ and laminin $(5 \mu \mathrm{g} / \mathrm{ml})$. Each chamber received $3 \times 10^{4}$ cells in $0.2 \mathrm{ml}$. After $1 \mathrm{~d}$ in vitro, the cells were fixed with buffered $4 \%$ paraformaldehyde for $30 \mathrm{~min}$ at $4^{\circ} \mathrm{C}$, rinsed with cold PBS, and processed for immunofluorescence.

Immunofluorescence. Slides were briefly boiled in $10 \mathrm{~mm}$ sodium citrate, $\mathrm{pH}$ 6.0, for antigen enhancement. Sections were treated with primary antibodies and fluorescent secondary antibodies as described previously (Hevner et al., 2004). To label DNA, sections were counterstained with $0.01 \%$ DAPI (4',6-diamidino-2-phenylindole) (Sigma, St. Louis, MO) or $0.1 \mu \mathrm{M}$ quinolinium, 4-[3-(3-methyl-2(3H)-benzothiazolylidene)-1propenyl]-1-[3-(trimethylammonio)propyl]-, diiodide (TO-PRO-3; Molecular Probes, Eugene, OR). For Tbr1/Tbr2 double labeling, sections were first processed for Tbrl by the ABC method (Vector Laboratories, Burlingame, CA) using diaminobenzidine as chromogen (Hevner et al., 2001) and then for Tbr2 immunofluorescence. The bright-field image of Tbr1 immunoreactivity was processed digitally using Photoshop (Adobe Systems, San Jose, CA) to invert brightness contrast and provide pseudocolor.

Primary antibodies. Mouse monoclonal antibodies were as follows: anti-BrdU (1:200), anti-neuronal-specific nuclear protein (NeuN) (1: 1500 ), and anti-proliferating cell nuclear antigen (PCNA) (1:2000) were from Chemicon (Temecula, CA); anti- $\beta$ III-tubulin (1:1000) was from Covance (Princeton, NJ); anti-nestin (rat-401; 1:50) and anti-Pax6 (1: 2000) were from Developmental Studies Hybridoma Bank (Iowa City, IA); anti-phosphovimentin (4A4; 1:1000) was from MBL International (Watertown, MA); and anti-Hu-C/D (1:50) was from Molecular Probes. Rat monoclonal antibody was anti-BrdU (1:200) from Harlan Sera-Lab (Loughborough, UK). Rabbit polyclonal antibodies were antiphosphohistone-H3 (1:400) from Upstate (Waltham, MA), and antiTbr1 (1:2000) and anti-Tbr2 (1:2000) from the laboratory of R.F.H.

Image processing, cell counting, and statistics. Epifluorescence was photographed digitally using a Spot Insight camera. Confocal images were acquired using a Bio-Rad (Hercules, CA) LS2000. Images were optimized for brightness and contrast using Photoshop. Cells were counted as described previously (Hevner et al., 2004). In vitro cell counts were pooled from two experiments, which used four or eight embryos each. In vivo cell counts were averaged from three age-matched embryos, except PCNA, which was studied in sections from only one embryo per age. For semiquantitative estimation of Pax6 and Tbr2 expression levels, fluorescence intensity was graded from 0 to +++ , as illustrated in supplemental Figure $2 B$ (available at www.jneurosci.org as supplemental material). Because mitotic figures never had fluorescence covering the entire nucleus, they were graded from 0 to ++ only.

\section{Results}

Previous work showed that, in the embryonic neocortex, Tbr2 mRNA is initially expressed in the preplate and subsequently in proliferative zones (Bulfone et al., 1999). We hypothesized that $\mathrm{Tbr}^{+}$cells in proliferative zones might be neuronal progenitor cells.
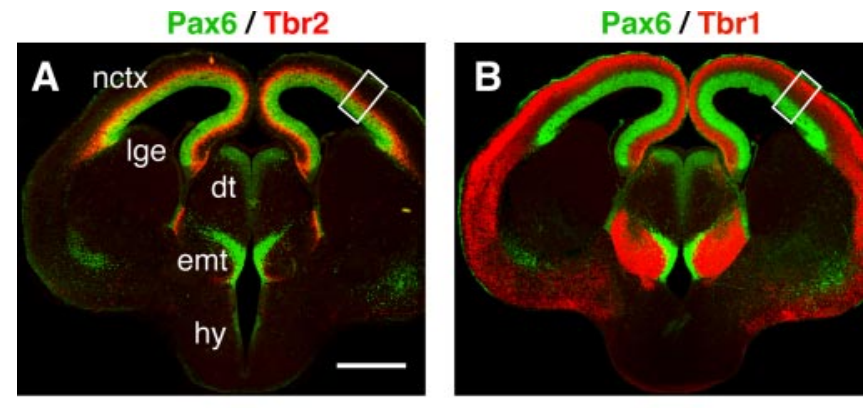

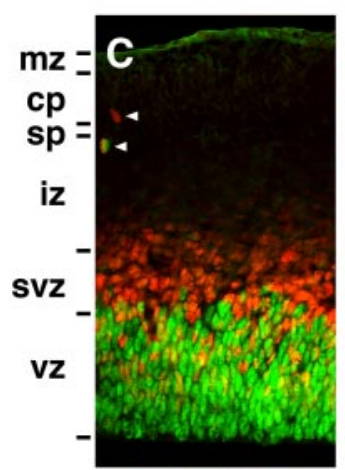

Pax6 / Tbr2

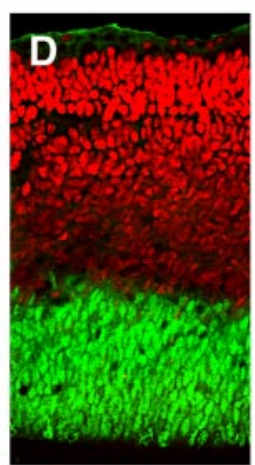

Pax6 / Tbr1

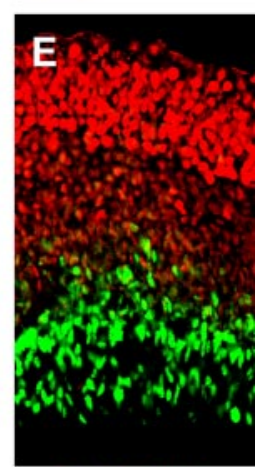

Tbr2/Tbr1
Figure 1. Expression of Pax6, Tbr2, and Tbr1 protein in E14.5 cortex (coronal sections). $A, C$ Pax6 (green) and Tbr2 (red). Boxed area in $A$ is shown at higher magnification in C. B, D, Pax6 (green) and Tbr1 (red). Boxed area in $B$ is shown at higher magnification in D.E, Tbr2 (green) and Tbr1 (red). All three TFs were expressed in the neocortex (nctx) and eminentia thalami (emt). Pax6 was also expressed in the ventricular zone of the dorsal thalamus (dt), hypothalamus (hy), and lateral part of the lateral ganglionic eminence (Ige). Within the neocortex, Pax6 was expressed in the ventricular zone (vz) only; Tbr2 was expressed in the ventricular zone, subventricular zone (svz), and intermediate zone (iz); and Tbr1 was expressed in the intermediate zone (iz), subplate (sp), cortical plate (cp), and marginal zone (mz). Rarely, Tbr2 ${ }^{+}$cells and Pax6 ${ }^{+}$ cells occupied superficial zones (arrowheads in C). Scale bar (in A): $A, B, 500 \mu \mathrm{m} ; C-E, 50 \mu \mathrm{m}$.

\section{Tbr2 protein is expressed in proliferative zones during cortical neurogenesis}

Immunofluorescence at different embryonic ages throughout neurogenesis showed that Tbr2 protein was expressed by two distinct sets of neocortical cells (supplemental Fig. 1, available at www.jneurosci.org as supplemental material). The first set to express Tbr2, from approximately E10.0 to E12.5, was differentiated preplate neurons, including reelin ${ }^{+}$Cajal-Retzius cells (supplemental Fig. $1 A-C$, available at www.jneurosci.org as supplemental material). The second set to express Tbr2, from approximately E11.5 to the end of neurogenesis, was immature cells in proliferative zones (supplemental Fig. $1 C-K$, available at www.jneurosci.org as supplemental material). The latter cells were most abundant from E13.5 to E16.5, during the peaks of neurogenesis and SVZ formation, and decreased markedly after E17.5, as neurogenesis slowed.

To define Tbr2 expression zones more precisely, we compared the distribution of Tbr $2^{+}$cells with Pax6 ${ }^{+}$cells and Tbr ${ }^{+}$cells in E14.5 cortex. Pax6 is expressed in the VZ by radial glia (Götz et al., 1998), and Tbrl is expressed in the IZ, subplate, cortical plate, and marginal zone by postmitotic neurons (Hevner et al., 2001). In these comparisons, Tbr $2^{+}$cells were concentrated mainly above the Pax6 ${ }^{+}$VZ (Fig. $1 A, C$ ) and below the Tbr ${ }^{+}$IZ (Fig. $1 B, E$ ), i.e., in the SVZ (Boulder Committee, 1970). In addition, many $\mathrm{Tbr}^{+}$cells were scattered in the basal VZ and lower IZ (Fig. 1C,E). Occasionally, isolated Tbr $2^{+}$cells were observed in unusual locations at the ventricular surface, in the upper IZ, in the subplate, or even in the cortical plate (Fig. $1 C$, arrowheads). A 
Tbr2 / nestin

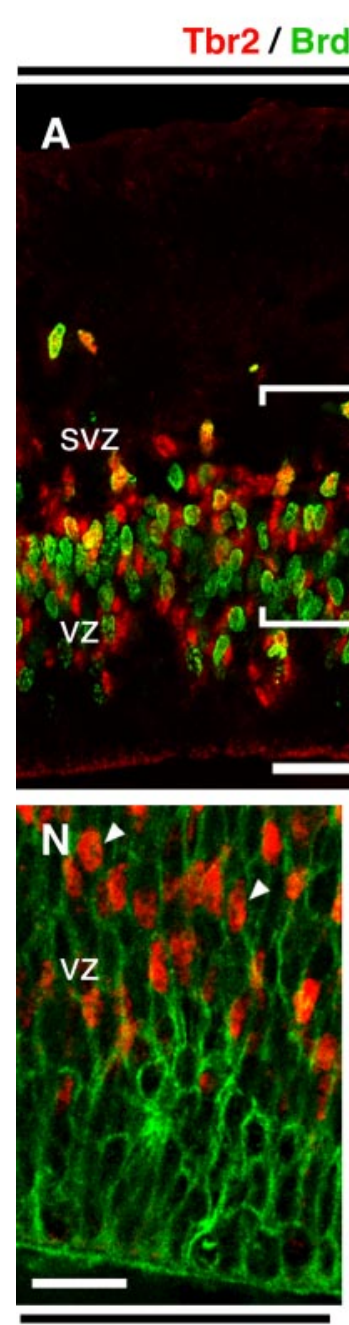

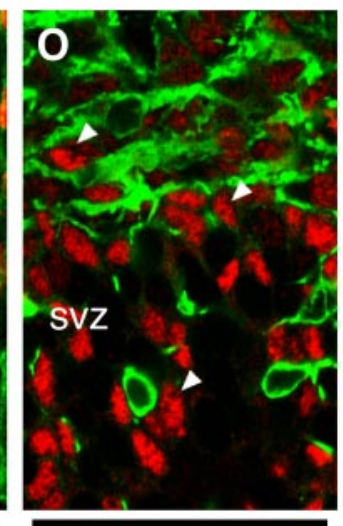

Tbr2 / $\beta$-tub
Tbr2 / Tbr2/
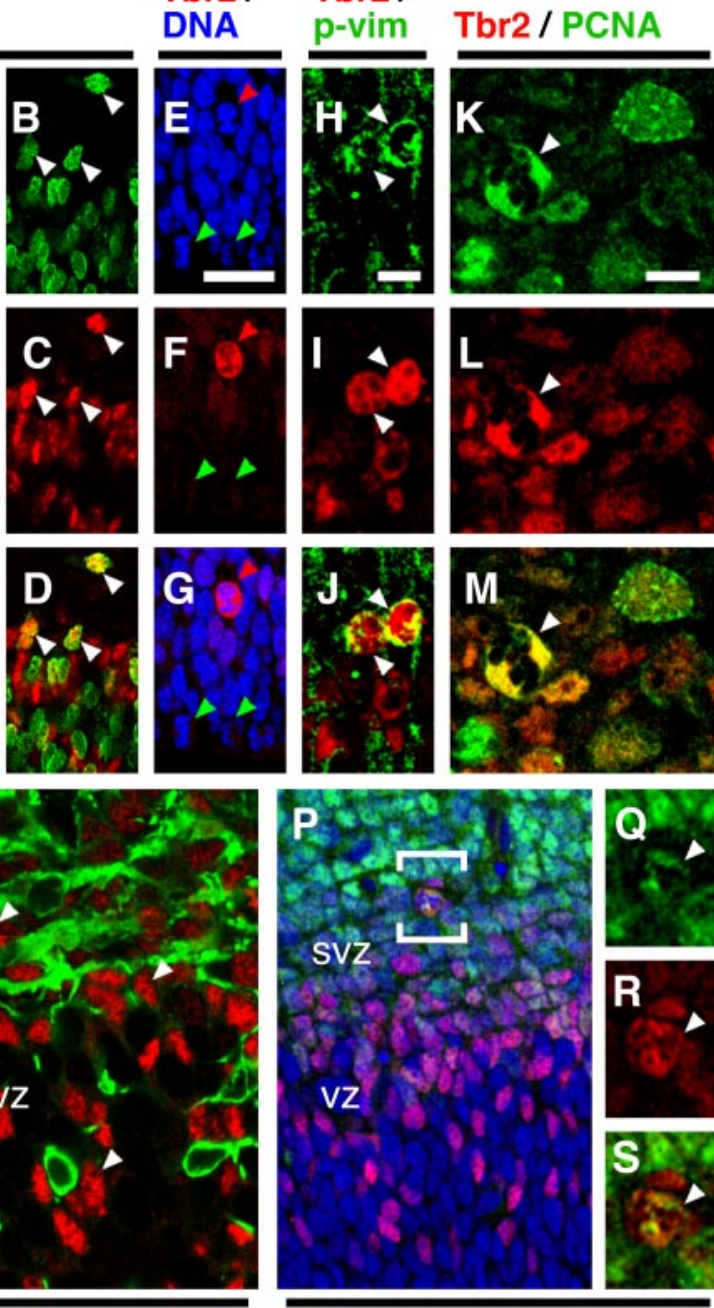

Tbr2 / NeuN / DNA
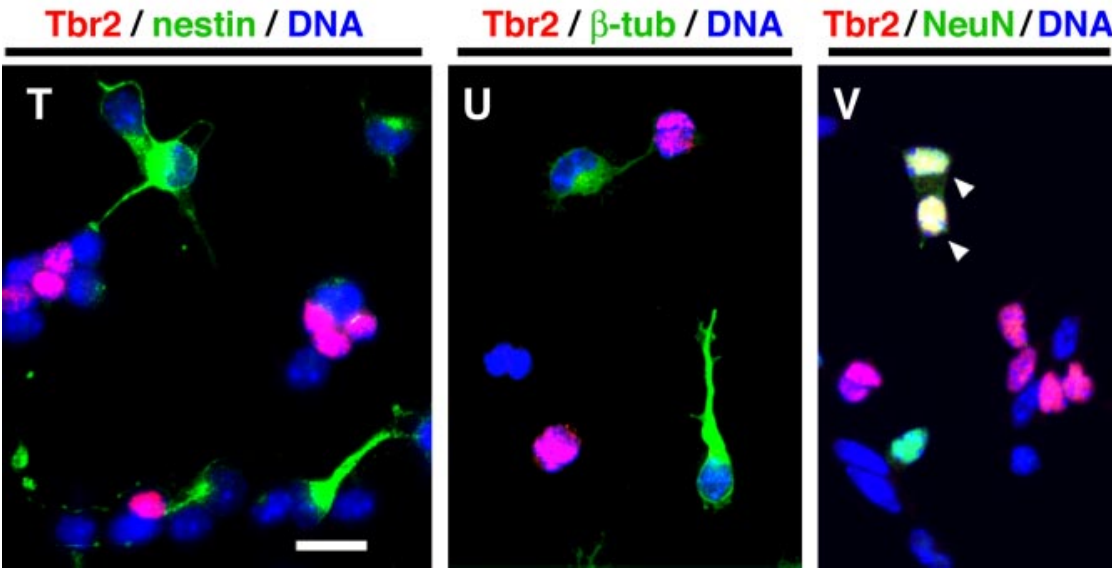

Figure 2. Tbr2 is expressed by NS-div IPCS. A-S, Confocal images of coronal sections through E14.5 cortex. A-D, Tbr2 (red) and BrdU (green). The bracketed area in $A$ is shown in separate color channels in $B-D$. Tbr2 was expressed by most $S$-phase cells in the SVZ and some in the VZ (arrowheads in B-D). E-G, Tbr2 (red) and DNA (blue). Tbr2 was expressed by NS-div cells (red arrowhead in $E-G$ ) but not by $S$-div cells (green arrowheads). $H-J$, Tbr2 (red) was expressed by phosphovimentin ${ }^{+}$(p-vim) M-phase cells (green) in the SVZ (arrowheads). $K-M$, Tbr2 (red) and PCNA (green). Most Tbr ${ }^{+}$cells contained PCNA, including mitotic figures (arrowheads). N, Tbr2 (red) and nestin (green). Most Tbr2 ${ }^{+}$cells (arrowheads) appeared to lack nestin. 0, Tbr2 (red) and $\beta$ IIItubulin ( $\beta$-tub; green). Most Tbr2 ${ }^{+}$cells (arrowheads) lacked $\beta$ III-tubulin. $P-S$, Tbr2 (red), NeuN (green), and DNA (blue). The bracketed area in $P$ is enlarged and shown in separate color channels in Q-S. Most Tbr $2^{+}$cells did not express NeuN, but those that did included some mitotic figures (arrowheads in Q-S). T-V, E14.5 cortical cells cultured for $1 \mathrm{~d}$ in vitro, labeled to detect Tbr2 (red), DNA (blue), and, in green, nestin $(T), \beta I l l-$ tubulin (U), or NeuN $(V)$. Few Tbr2 ${ }^{+}$cells expressed nestin (5.1\%) or $\beta$ III-tubulin (12.7\%), but more expressed NeuN (20.8\%; arrowheads). Scale bars: (in $A) A-D, P, 50 \mu \mathrm{m}$; (in $E) E-G, 20 \mu \mathrm{m}$; (in $H$ ) $H-J, Q-S, 10$ $\mu \mathrm{m}$; (in K) K-M, 0, $10 \mu \mathrm{m}$; (in $N$ ) $N, 20 \mu \mathrm{m}$; (in $T) T-V, 20 \mu \mathrm{m}$. high lateral to low medial gradient of Tbr2 protein expression was noted, similar to the gradient of mRNA expression (Bulfone et al., 1999).

The locations of $\mathrm{Tbr}^{+}$cells in the basal VZ, SVZ, and lower IZ appeared similar to the distribution of NS-div cells or IPCs, as reported previously (Smart, 1973; Haubensak et al., 2004; Miyata et al., 2004; Noctor et al., 2004).

\section{Tbr2 is expressed by}

\section{neurogenic progenitors}

To determine whether Tbr2 ${ }^{+}$cells are mitotically active, we tested for Tbr 2 colocalization with four markers of mitotic activity: (1) acute BrdU incorporation, an S-phase marker; (2) condensed chromatin, an M-phase marker; (3) phosphovimentin, another M-phase marker (Kamei et al., 1998); and (4) PCNA, which is not specific to any particular phase of the mitotic cycle (McKeever, 1998).

Acute BrdU incorporation in E14.5 cortex showed that Tbr2 was expressed by many S-phase cells in the SVZ and some in the VZ (Fig. $2 A-D$ ). Overall, $\sim 19.3 \pm$ $1.9 \%($ mean $\pm \mathrm{SD})$ of all $\mathrm{BrdU}^{+}$cells $(n=$ 900) expressed Tbr2. Notably, most $\mathrm{BrdU}^{+}$cells in the SVZ expressed Tbr2, but most in the VZ did not (Fig. 2A-D). A relation between cell location and Tbr2 expression was also observed for M-phase cells: NS-div cells almost always expressed Tbr2, but surface-dividing (S-div) cells almost never did (Fig. $2 E-M$ ). This relation held true throughout early, middle, and late neurogenesis. In E12.5 cortex, Tbr2 was expressed by $100 \pm 0 \%$ of NS-div cells $(n=47)$ and $3.2 \pm 2.1 \%$ of S-div cells $(n=$ 317). In E14.5 cortex, Tbr2 was expressed by $98 \pm 1.9 \%$ of NS-div cells $(n=92)$ and $1.2 \pm 2.4 \%$ of S-div cells $(n=495)$. In E16.5 cortex, Tbr2 was expressed by $98 \pm$ $3.4 \%$ of NS-div cells $(n=46)$ and $1.1 \pm$ $2.0 \%$ of S-div cells $(n=191)$. The ratio of NS-div mitoses to all mitotic figures increased gradually during neurogenesis, from $12.9 \pm 1.8 \%$ on E12.5, to $15.7 \pm$ $1.9 \%$ on E14.5, to $19.4 \pm 6.5 \%$ on E16.5. PCNA expression (Fig. $2 K-M$ ) suggested that most Tbr ${ }^{+}$cells were mitotically active $(94 \% ; n=183)$. Because NS-div cells and S-div cells generally correspond to IPCs and radial glia, respectively (Haubensak et al., 2004; Miyata et al., 2004; Noctor et al., 2004), these data suggested that Tbr2 is specifically expressed by IPCs and not radial glia.

To further characterize Tbr $2^{+}$cells, we studied markers of differentiation status. Nestin, an intermediate filament protein expressed by many progenitor cells, ap- 
peared not to be expressed by most Tbr $2^{+}$cells in E14.5 cortex (Fig. $2 N$ ). Because the high density of nestin ${ }^{+}$processes made colocalization difficult to assess in tissue sections, we also studied dissociated cell cultures. This approach indicated that nestin was expressed by only $\sim 5.1 \%$ of Tbr $2^{+}$cells $(n=78)$ in vitro (Fig. $2 T)$. To evaluate possible neuronal differentiation, we studied three neuronal markers: $\beta$ III-tubulin, NeuN, and Hu-C/D. $\beta$ IIITubulin was expressed by few Tbr2 ${ }^{+}$cells in vivo (Fig. 2O) and $12.7 \%$ of Tbr $2^{+}$cells $(n=110)$ in vitro (Fig. $\left.2 U\right)$. NeuN was generally not expressed in the VZ and SVZ and thus was not expressed by most $\mathrm{Tbr} 2^{+}$cells (Fig. $2 P$ ) (see also supplemental Fig. $1 H$, available at www.jneurosci.org as supplemental material). However, NeuN was expressed weakly by some $\mathrm{Tbr} 2^{+}$cells in the SVZ and lower IZ (Fig. $2 P-S$ ), including most Tbr2 ${ }^{+}$ mitotic figures $(57.3 \pm 15.8 \% ; n=96)$. In vitro, $20.8 \%$ of Tbr $2^{+}$ cells $(n=125)$ expressed NeuN (Fig. $2 V)$. Hu-C/D was also detected in some Tbr ${ }^{+}$NS-div cells in vivo (supplemental Fig. $1 C-G$, available at www.jneurosci.org as supplemental material). These neuronal markers suggested that most Tbr2 ${ }^{+}$cells were committed to neuronal fate by the time of M-phase. The $\mathrm{Tbr} 2^{+} /$ $\mathrm{NeuN}^{+}$cells were interpreted as differentiating neurons, completing the transition from IPCs. Finally, $100 \%$ of $\mathrm{Tbr}^{+}{ }^{+}$postmitotic neurons $(n=130)$ expressed $\mathrm{NeuN}$ in vivo (data not shown).

\section{Pax6 is downregulated in most Tbr ${ }^{+}$IPCs}

Previous work has shown that Pax6 is expressed by radial glia but not by progenitors in the SVZ (Götz et al., 1998). Because Tbr2 demonstrates the opposite pattern, we studied Pax6 ${ }^{+}$cells for direct comparison. Acute BrdU labeling showed that most S-phase cells in the VZ expressed Pax6, but S-phase cells in the SVZ did not (Fig. 3A-D). Among cells in M-phase, marked by chromatin condensation or phosphohistone-H3 expression (Estivill-Torrus et al., 2002), Pax6 was expressed strongly by S-div cells but weakly or not at all by NS-div cells (Fig. 3E). This relation also held true in E12.5, E14.5, and E16.5 cortex (supplemental Fig. 2A, available at www.jneurosci.org as supplemental material). These results reinforced the impression that Pax6 was expressed in different progenitor cells (S-div) than Tbr2 (NS-div).

Pax6/Tbr2 double immunofluorescence and confocal imaging revealed that most progenitor cells expressed only Pax6 or only Tbr2, although a significant minority expressed both TFs (Fig. 3F-I). Of 1989 cells immunoreactive for Pax6 or Tbr2 in E14.5 cortex, $55.7 \pm 9.9 \%$ were Pax6 ${ }^{+}$only, $32.8 \pm 10.2 \%$ were $\mathrm{Tbr}^{+}$only, and $11.5 \pm 1.0 \%$ were Pax $6^{+} / \mathrm{Tbr} 2^{+}$. In most cells in which Pax6 and Tbr2 were coexpressed, neither TF reached high levels (supplemental Fig. $2 B$, available at www.jneurosci.org as supplemental material). The $\mathrm{Pax} 6^{+} / \mathrm{Tbr} 2^{+}$cells were interpreted as differentiating IPCs $\left(\right.$ Tbr $2^{+}$), newly derived from radial glia $\left(\mathrm{Pax}^{+}\right)$. Tbr2/Pax6 double immunofluorescence also confirmed that S-div mitoses expressed Pax6 but not Tbr2 (Fig. 3I, arrowheads), whereas NS-div mitoses expressed Tbr 2 but little or no Pax6 (Fig. 3H, arrowhead).

\section{Discussion}

Our results suggest that Tbr2 is expressed by a subset of cortical progenitor cells, which are distinct from radial glia, and have properties of IPCs (most importantly, non-surface mitosis). The other major conclusion is that Pax6, Tbr2, and Tbr1 are expressed sequentially in the neurogenesis of glutamatergic neurons from radial glia and IPCs.
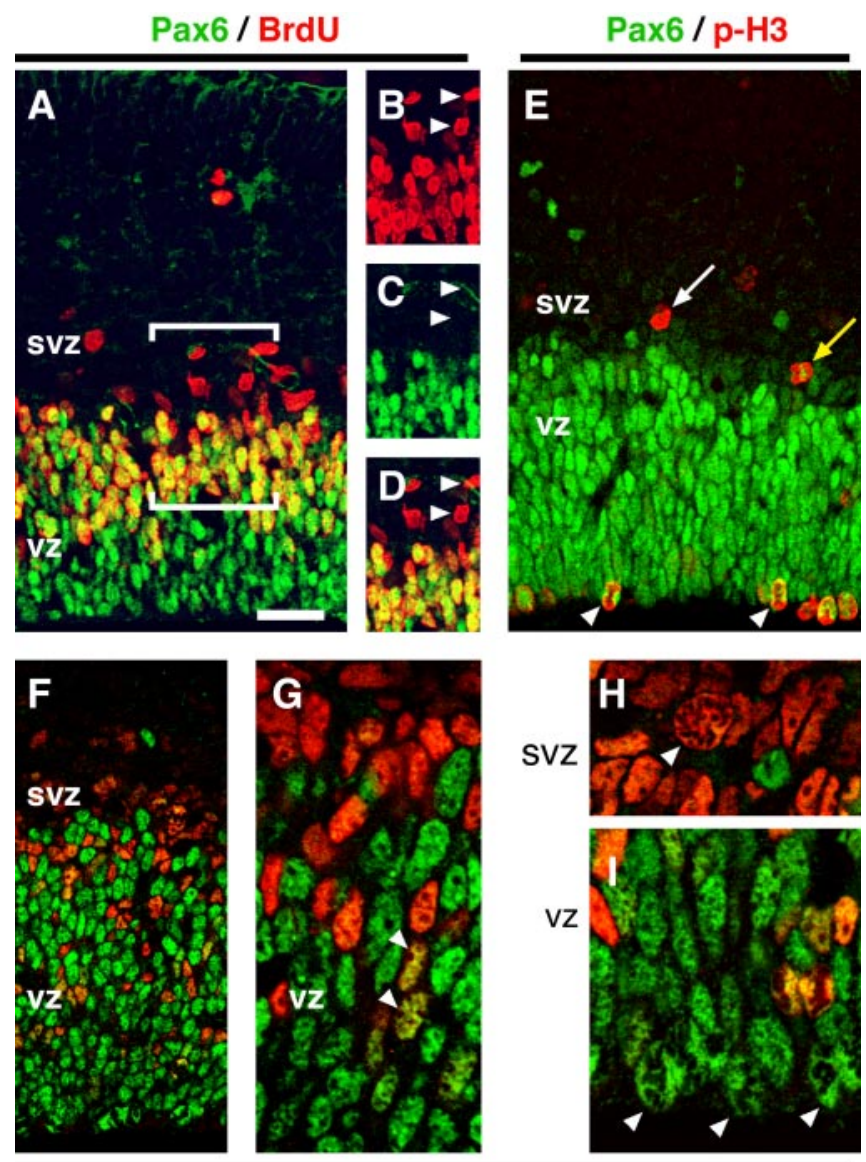

Pax6 / Tbr2

Figure 3. Pax6 is substantially downregulated in most Tbr2 ${ }^{+}$IPCs. A-I, Confocal images of coronal sections through E14.5 cortex. A-D, Pax6 (green) and BrdU (red). The bracketed area in $A$ is shown in separate color channels in $B-D$. Pax6 was not expressed by most $S$-phase cells in the SVZ (arrowheads in $B-D$ ) but was expressed by most S-phase cells in the VZ. E, Pax6 (green) and phosphohistone-H3 (red). Pax6 was strongly expressed in S-div mitotic figures (arrowheads) but only weakly (yellow arrow) or not at all (white arrow) in NS-div cells. F-I, Pax6 (green) and Tbr2 (red) double immunofluorescence. Most VZ and SVZ cells expressed Pax6 or Tbr2 but not both. Double-labeled cells appeared yellow (arrowheads in G). $H-I$, Higher magnification of mitotic figures in the SVZ $(H)$ and VZ $(I)$. NS-div mitoses were predominantly $\mathrm{Tbr}^{+}$(arrowhead in $H$ ), and S-div mitoses were Pax6 ${ }^{+}$(arrowheads in $I$ ). Scale bar (in $A$ ): $A-E, 30 \mu \mathrm{m} ; F, 40 \mu \mathrm{m} ; G-I, 12 \mu \mathrm{m}$.

\section{IPC characteristics of $\mathrm{Tbr} 2^{+}$cells}

Progenitor cells that expressed Tbr2 had several characteristics of IPCs, as defined in recent studies (Haubensak et al., 2004; Miyata et al., 2004; Noctor et al., 2004). First, Tbr2 was expressed in NS-div mitotic figures, most of which ( $\sim 90 \%$ or more) are neurogenic IPCs, at least during early and middle stages of corticogenesis. Second, Tbr2 expression in proliferative zones rose and fell with cortical plate neurogenesis (supplemental Fig. 1, available at www.jneurosci.org as supplemental material). Third, many $\mathrm{Tbr}^{+}{ }^{+}$mitotic figures $(57.3 \%)$ expressed NeuN in vivo, suggesting their neurogenic commitment (Fig. $2 P-S$ ). Fourth, a significant minority of Tbr ${ }^{+}$cells coexpressed Pax6, consistent with an origin from cortical radial glia, as reported for IPCs. The only discrepancy was that, in contrast to IPCs (Miyata et al., 2004; Noctor et al., 2004), most Tbr2 ${ }^{+}$cells did not appear to express nestin (Fig. $2 N, T$ ). This could be a technical difference.

Our data suggest that Tbr2 is expressed throughout the IPC "life cycle." Presumably, new IPCs inherit Pax6 passively from radial glia, accounting for low-level Pax6 expression in some 
Tbr $2^{+}$cells. Tbr2 was highly expressed during S-phase (Fig. $2 A-D$ ) and $M$-phase (Fig. $2 E-M$ ) of IPCs. Interestingly, Tbr2 ${ }^{+}$ cells divided not only in the SVZ but also frequently in the basal $\mathrm{VZ}$ and less often in the IZ. These locations matched the distribution of NS-div cells as documented by Smart (1973). Tbr2 expression continued into the beginning of neuronal differentiation, as indicated by NeuN immunoreactivity in some $\mathrm{Tbr} 2^{+}$ cells (Fig. $2 P-S, V$ ). Finally, Tbr2 was downregulated as immature neurons migrated through the IZ toward the cortical plate and concurrently upregulated Tbr1 (Fig. 1).

Most Tbr ${ }^{+}$cells in the VZ and SVZ are probably progenitor cells, as inferred from 94\% PCNA coexpression (Fig. $2 \mathrm{~K}-\mathrm{M}$ ). However, Tbr2 is not completely specific for IPCs, because it was also expressed by early postmitotic neurons derived from IPCs (Fig. $2 P, V$ ). It is unknown whether neurons derived directly from radial glia express Tbr2 at any time. Such cells may remain in the VZ for 10 hr or more after mitosis (Noctor et al., 2004) and could express Tbr2 during that time. Also, the properties of NSdiv progenitors in late corticogenesis (E15.5-E17.5) have not been studied much, and they might produce glia, as well as neurons. Along this line, a few Tbr ${ }^{+}$cells persisted in the VZ/SVZ postnatally (supplemental Fig. $1 K$, available at www.jneurosci.org as supplemental material). Tbr ${ }^{+}$cells appear to originate mainly from cortical radial glia, as evidenced by Pax6 coexpression (Fig. $3 F-I$ ), but additional sources (possibly extracortical) cannot be ruled out yet.

\section{Pax6, Tbr2, and Tbr1 functions in cortical neurogenesis}

The sequential expression of Pax6, Tbr2, and Tbr1 suggests that each TF regulates discrete steps in projection neuron differentiation. Pax6 appears to be most important for production and glutamatergic differentiation of late-born neurons (Schuurmans et al., 2004) and for neurogenesis in the SVZ, which is abnormal in Pax6-deficient (Sey/Sey) mice (Tarabykin et al., 2001). The SVZ defect in Sey/Sey cortex could suggest that IPC development is impaired. However, there is no apparent decrease of "nonradial glia neurogenic progenitors" (presumably IPCs) in E14 Sey/Sey cortex (Heins et al., 2002), and NS-div mitoses may actually increase in Sey/Sey cortex (Estivill-Torrus et al., 2002). Tbr2 mRNA expression is overall reduced in Sey/Sey lateral neocortex, but this could be a defect of regional specification (Muzio et al., 2002). The data seem to suggest that Pax6 regulates regional and kinetic aspects of IPC production, as well as glutamatergic differentiation.

The homology between Tbr2 and Tbr1, and their sequential expression in the glutamatergic lineage, suggest that they may have related functions, although any interactions are still unknown. Tbr1 knock-out causes defects of cortical lamination, axon connections, and layer-specific differentiation (Hevner et al., 2001). Tbr2 knock-out causes early embryonic lethality, before cortex formation (Russ et al., 2000). The expression of Tbr2 in IPCs suggests that Tbr2 may regulate early steps in projection neuron fate commitment or differentiation. In future studies, we plan to determine the proportion of cortical neurons in each layer that are derived from $\mathrm{Tbr} 2^{+}$cells and to study mutant mice in which Tbr2 gene disruption is limited to the CNS only.

\section{References}

Boulder Committee (1970) Embryonic vertebrate central nervous system: revised terminology. Anat Rec 166:257-261.

Bulfone A, Smiga SM, Shimamura K, Peterson A, Puelles L, Rubenstein JLR
(1995) T-brain-1: a homolog of Brachyury whose expression defines molecularly distinct domains within the cerebral cortex. Neuron 15:63-78.

Bulfone A, Martinez S, Marigo V, Campanella M, Basile A, Quaderi N, Gattuso C, Rubenstein JLR, Ballabio A (1999) Expression pattern of the Tbr2 (Eomesodermin) gene during mouse and chick brain development. Mech Dev 84:133-138.

Estivill-Torrus G, Pearson H, van Heyningen V, Price DJ, Rashbass P (2002) Pax6 is required to regulate the cell cycle and the rate of progression from symmetrical to asymmetrical division in mammalian cortical progenitors. Development 129:455-466.

Götz M, Stoykova A, Gruss P (1998) Pax6 controls radial glia differentiation in the cerebral cortex. Neuron 21:1031-1044.

Haubensak W, Attardo A, Denk W, Huttner WB (2004) Neurons arise in the basal neuroepithelium of the early mammalian telencephalon: a major site of neurogenesis. Proc Natl Acad Sci USA 101:3196-3201.

Heins N, Malatesta P, Cecconi F, Nakafuku M, Tucker KL, Hack MA, Chapouton P, Barde Y-A, Götz M (2002) Glial cells generate neurons: the role of the transcription factor Pax6. Nat Neurosci 5:308-315.

Hevner RF, Shi L, Justice N, Hsueh Y-P, Sheng M, Smiga S, Bulfone A, Goffinet AM, Campagnoni AT, Rubenstein JLR (2001) Tbrl regulates differentiation of the preplate and layer 6. Neuron 29:353-366.

Hevner RF, Daza RAM, Englund C, Kohtz J, Fink A (2004) Postnatal shifts of interneuron position in the neocortex of normal and reeler mice: evidence for inward radial migration. Neuroscience 124:605-618.

Kamei Y, Inagaki N, Nishizawa M, Tsutsumi O, Taketani Y, Inagaki M (1998) Visualization of mitotic radial glial lineage cells in the developing rat brain by cdc2 kinase-phosphorylated vimentin. Glia 23:191-199.

Malatesta P, Hartfuss E, Götz M (2000) Isolation of radial glial cells by fluorescent-activated cell sorting reveals a neuronal lineage. Development 127:5253-5263.

Malatesta P, Hack MA, Hartfuss E, Kettenmann H, Klinkert W, Kirchhoff F, Götz M (2003) Neuronal or glial progeny: regional differences in radial glia fate. Neuron 37:751-764.

McKeever PE (1998) Insights about brain tumors gained through immunohistochemistry and in situ hybridization of nuclear and phenotypic markers. J Histochem Cytochem 46:585-594.

Miyata T, Kawaguchi A, Okano H, Ogawa M (2001) Asymmetric inheritance of radial glial fibers by cortical neurons. Neuron 31:727-741.

Miyata T, Kawaguchi A, Saito K, Kawano M, Muto T, Ogawa M (2004) Asymmetric production of surface-dividing and non-surface-dividing cortical progenitor cells. Development 131:3133-3145.

Muzio L, DiBenedetto B, Stoykova A, Boncinelli E, Gruss P, Mallamaci A (2002) Conversion of cerebral cortex into basal ganglia in $E m \times 2^{-1-}$ Pax6 $^{\text {Sey/Sey }}$ double-mutant mice. Nat Neurosci 5:737-745.

Noctor SC, Flint AC, Weissman TA, Dammerman RS, Kriegstein AR (2001) Neurons derived from radial glial cells establish radial units in neocortex. Nature 409:714-720.

Noctor SC, Martínez-Cerdeño V, Ivic L, Kriegstein AR (2004) Cortical neurons arise in symmetric and asymmetric division zones and migrate through specific phases. Nat Neurosci 7:136-144.

Russ AP, Wattler S, Colledge WH, Aparicio SAJR, Carlton MBL, Pearce JJ, Barton SC, Surani MA, Ryan K, Nehls MC, Wilson V, Evans MJ (2000) Eomesodermin is required for mouse trophoblast development and mesoderm formation. Nature 404:95-99.

Schuurmans C, Armant O, Nieto M, Stenman JM, Britz O, Klenin N, Brown C, Langevin L-M, Seibt J, Tang H, Cunningham JM, Dyck R, Walsh C, Campbell K, Polleux F, Guillemot F (2004) Sequential phases of cortical specification involve Neurogenin-dependent and -independent pathways. EMBO J 23:2892-2902.

Smart IHM (1973) Proliferative characteristics of the ependymal layer during the early development of the mouse neocortex: a pilot study based on recording the number, location, and plane of cleavage of mitotic figures. J Anat 116:67-91.

Tarabykin V, Stoykova A, Usman N, Gruss P (2001) Cortical upper layer neurons derive from the subventricular zone as indicated by Svet1 gene expression. Development 128:1983-1993.

Xu Q, Cobos I, De La Cruz E, Rubenstein JL, Anderson SA (2004) Origins of cortical interneuron subtypes. J Neurosci 24:2612-2622. 